\title{
Feathers fly in Beijing
}

\author{
The question of whether birds evolved from dinosaurs arouses strong \\ opinions. Rex Dalton reports on a scientific meeting that at times bore \\ more resemblance to a political sparring match.
}

W hen ornithologists and palaeontologists rolled into Beijing earlier this month for the fifth quadrennial meeting of the Society of Avian Paleontology and Evolution, it seemed like a perfect opportunity to try and resolve the longrunning debate over whether birds evolved from dinosaurs.

Northeast China has produced a wealth of bird and dinosaur fossils in recent years, and these have been used to explore the links between the two groups. At the meeting, Chinese scientists showed off some newly discovered specimens, which might help to answer important evolutionary questions.

But by the close of the meeting, hosted by Beijing's Institute of Vertebrate Paleontology and Paleoanthropology (IVPP), the divisions between those who believe birds evolved from dinosaurs and those who disagree appeared greater than ever. Several attendees were disappointed that progressive scientific debate was stifled by entrenched opinions, raised voices and strident words. "I think a lot more interesting issues could have come up, instead of going over the same old tired stories, like the origin of flight," says John Hutchinson, a doctoral student at the University of California at Berkeley. "It is like the field hasn't moved on."

"The time is now to stop debating and proceed with learning about the biology of the origin of birds," agrees Richard Prum, an evolutionary biologist at the University of Kansas in Lawrence. But when Prum argued at the meeting that birds' feathers have the same

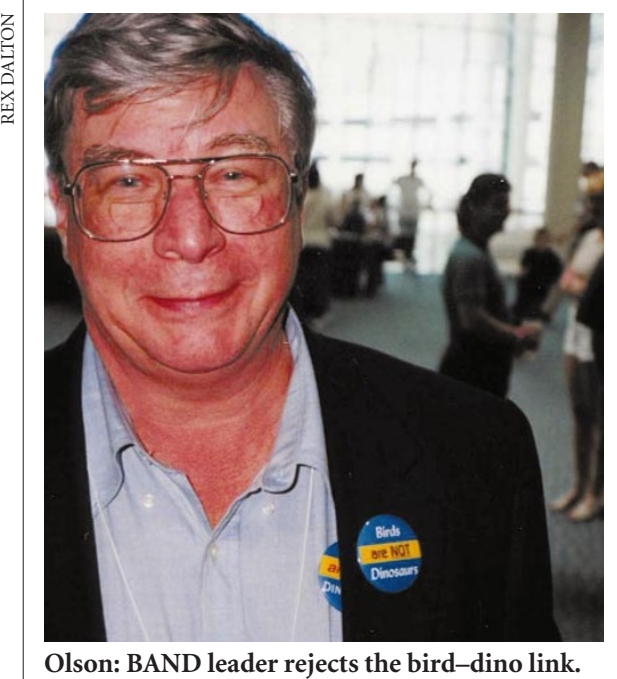

Olson: BAND leader rejects the bird-dino link.

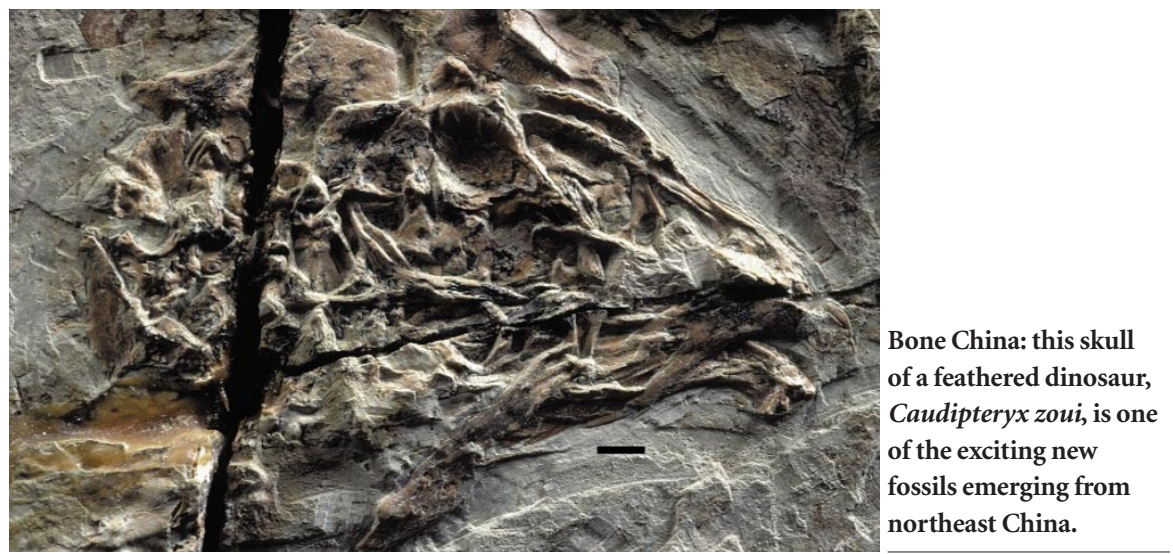

evolutionary origin as the hair-like integumentary filaments seen on many dinosaur fossils, he was accused by Storrs Olson, head of ornithology at the National Museum of Natural History in Washington DC, of engaging in "ideological mumbo jumbo".

Olson is a leader in the camp that believes that birds evolved separately from dinosaurs. He rejects the phylogenetic analysis used by Prum and others to build evolutionary links between birds and dinosaurs on the basis of shared characteristics. Throughout the meeting, Olson and like-minded scientists wore badges saying "Birds are Not Dinosaurs".

But this 'BAND' group was not alone in adopting tactics that had more in common with political point-scoring than scientific discourse. When Alan Feduccia, a palaeobiologist at the University of North Carolina at Chapel Hill, gave a talk on why the theory of bird evolution from dinosaurs should be rejected, his argument was likened by some opponents to those of creationists. Chris McGowan, curator of palaeobiology at the Royal Ontario Museum in Toronto, said cuttingly that he had not enjoyed such a performance since he last heard a talk by Duane Gish, senior vice-president of the Institute for Creation Research in Santee, near San Diego.

But between such acrimonious exchanges, new lines of research did emerge. Mary Schweitzer of Montana State University in Bozeman described how she has looked for evidence of feathers on a Mongolian dinosaur fossil, Shuvuuia deserti, using fluorescently labelled antibodies for the protein keratin. Schweitzer detected $\beta$-keratin, the basic component of most feathers, but significantly she found no evidence of $\alpha$-keratin, which does not occur in feathers.
Hutchinson, who studies at Berkeley with Kevin Padian, a leading proponent of the idea that birds evolved from dinosaurs, presented data on hind-limb evolution. Hutchinson compares bones of fossils and existing species for scarring to determine where muscles attach. He uses this information to generate a computer model of the biomechanics of hind limbs. As an example, he presented preliminary data on how much hind-limb muscle mass would be needed to support a Tyrannosaurus rex. Hutchinson hopes that the modelling process will be useful for exploring the links between birds and dinosaurs.

Kevin Middleton, a doctoral student in evolutionary biology at Brown University in Providence, Rhode Island, described his studies of the feet of birds and dinosaurs. $\mathrm{He}$ has focused on the evolution of the first digit, or hallux, the orientation of which is used to help classify species as birds or dinosaurs. The bone is reversed in perching birds, but Middleton has produced evidence that it is not only reversed, but also rotated along its axis. "I'm fairly confident the hallux is much more complex than we thought," says Middleton.

"These are examples of the next generation of research that will provide tremendous progress," says Prum. And the specimens being uncovered in northeast China are likely to be key to this progress. During the meeting, one IVPP scientist, Fucheng Zhang, presented tantalizing slides of about half a dozen undescribed species.

But if the Beijing meeting is anything to go by, any resolution of the debate over the origin of birds may take years to emerge. When the proponents get together in the same room, they seem to generate more heat than light. Rex Dalton is Nature's West Coast US Correspondent. 